\title{
MATRIX D.G. NEAR-RINGS
}

\author{
by V. THARMARATINAM
}

(Received 21st May 1996)

\begin{abstract}
Matrix near-rings had been defined by Meldrum and Van der Walt in 1986 and although a fair amount of results on the structure of these near-rings have been obtained since then, a satisfactory structure theory has yet to be developed for matrix d.g. near-rings. In this paper we give an alternate definition (in fact the dual definition) for matrix d.g. near-rings and develop a satisfactory structure theory for such d.g. near-rings.
\end{abstract}

1991 Mathematics subject classification: 16 Y 30.

\section{Introduction}

In the study of near-rings one would like to have the analogue of matrix rings. A natural choice would be the system $M_{n}(R)$ of all matrices having entries from a nearring $R$ together with the normal operations of matrix addition and multiplication. But unfortunately the multiplication is not necessarily associative and thus, in general, $M_{n}(R)$ is not a near-ring.

Beidleman [4] has shown that if $R$ is a near-ring with identity and for some integer $n(>1)$ we have $M_{n}(R)$ to be a near-ring, then $R$ is a ring. Ligh [10] has shown that when $n>1, M_{n}(R)$ is a near-ring if and only if $R$ is $n$-distributive. Thus $M_{n}(R)$ as defined above fails to be the near-ring (or d.g. near-ring) analogue of matrix rings.

Meldrum and Van der Walt [14] defined the matrix near-ring over a near-ring $R$ as the sub near-ring of $\operatorname{Map}\left(R^{n}, R^{n}\right)$ generated by the set $\left\{f_{i j}^{r}: R^{n} \rightarrow R^{n} \mid r \in R, 1 \leq i, j \leq n\right\}$ of maps, which in the ring case correspond to the matrices with $r$ in the $(i, j)$ th position and zero elsewhere. A fair amount of results on the structure of these near-rings had been obtained in $[1,2,3,12,13,14,15,22$ and 23]. However, in our view, a satisfactory structure theory has yet to be developed for matrix d.g. near-rings and we present in this paper an alternate definition (in fact the dual definition) for matrix d.g. near-rings and develop a satisfactory structure theory for such d.g. near-rings.

Meldrum and Van der Walt [14] took the view that an $n \times n$ matrix over a ring $R$ may also be considered as an endomorphism of the abelian group $R^{n}$ (where $R^{n}$ denotes the direct sum of $n$ copies of $(R,+))$ and their matrices over a near-ring $R$ are maps from $R^{n}$ to $R^{n}$. We start with the characterisation of an $n \times n$ matrix over a ring $R$ as an $R$ endomorphism of a free $R$-module of rank $n$ and we characterise an $n \times n$ matrix over a d.g. near-ring $R$, distributively generated by a semigroup $S$, as an $R$-endomorphism of a $v(R,+)$ - free left $(R, S)$-group $\Omega$ on a base with $n$ elements; here $v(R,+)$ denotes the variety 
of left $(R, S)$-groups generated by the left $(R, S)$-group $(R,+)$. We have shown in [18] that the set of all such $R$-endomorphisms forms a d.g. near-ring and our matrices have been defined in such a manner as to ensure that our matrix d.g. near-ring is near-ring isomorphic to the above endomorphism d.g. near-ring. Further our non-singular matrices correspond to the $R$-automorphisms of $\Omega$ in this isomorphism.

Thus our matrix d.g. near-rings are Neumann d.g. near-rings (named after Hanna Neumann for her work in [16]) but not conversely. It may be observed that Hanna Neumann in [16] had, in fact, commented on the similarity of her near-rings to ordinary matrix rings.

We define an $m \times n$ matrix over a d.g. near-ring $R$ as a column vector having $m$ rows with an $R$-word in $n$ variables in each row; an $R$-word $w\left(x_{1}, \ldots, x_{n}\right)$ is defined to be zero if $w\left(r_{1}, \ldots, r_{n}\right)=0$ for all $r_{1}, \ldots, r_{n}$ in $R$ and matrix multiplication is by substitution of the variables. Historically, matrices originated from systems of linear equations and matrix multiplication from substitution of the variables. Thus our definition is a very natural generalisation and in the case when $R$ is a ring we get the usual $m \times n$ matrix over $R$.

In Section 3 we give our definition of matrices over a d.g. near-ring and in Section 4 we obtain, in particular, generalisations of the Wedderburn-Artin Theorem for rings and the Morita criterion for equivalence of the rings $R$ and $M_{n}(R)$.

In Section 5 we develop the theory of dual $(R, S)$-groups and prove that the $R^{n}$ utilised by Meldrum and Van der Walt in their definition of matrix near-rings is the dual left $(R, S)$-group of our $v(R,+)$-free left $(R, S)$-group $\Omega$ and that our matrix d.g. near-ring is near-ring isomorphic to the matrix d.g. near-ring defined by Meldrum and Van der Walt.

\section{Preliminaries and definitions}

Throughout this paper we will assume (i) the term near-ring refers to a right nearring with identity, (ii) $R$ is an abstract d.g. near-ring with identity $e, D(R)$ is the set of distributive elements in $R, S$ is a distributive semigroup generating $(R,+)$ and that 0 and $e$ are in $S$, (iii) $v(R,+)$ denotes the variety of left $(R, S)$-groups generated by $(R,+)$, (iv) the basic definitions in [5], [17] and [18], (v) $n$ is an arbitrary natural number, (vi) Capital Roman letters signify near-rings and their subsets or matrices and rows of matrices and small Roman letters signify the elements or near-rings, (vii) Capital Greek letters stand for groups or their subsets and small Greek letters for elements of groups or maps.

Definition 2.1. A right $R$-group is an additive group $\Omega$ together with a $\operatorname{map}(\omega, x) \rightarrow \omega x$ of $\Omega \times R \rightarrow \Omega$ such that

(i) $\left(\omega_{1}+\omega_{2}\right) x=\omega_{1} x+\omega_{2} x$ for all $\omega_{1}, \omega_{2} \in \Omega$ and $x \in R$;

(ii) $\omega(x y)=(\omega x) y$ for all $\omega \in \Omega$ and $x, y \in R$;

(iii) $\omega e=\omega$ for all $\omega \in \Omega$. 
Definition 2.2. An element $\lambda \in \Omega$ is said to be distributive if $\lambda(x+y)=\lambda x+\lambda y$ for all $x, y \in R$.

The set $D(\Omega)$ of all distributive elements in $\Omega$ is non-empty as $0_{\Omega} \in D(\Omega)$ and by Proposition 1.2 of $[18]$ we have $D(\Omega) D(R) \subseteq D(\Omega)$.

Definition 2.3. A d.g. right $(R, S)$-group is a group $\Omega$ such that (i) $\Omega$ is a right $R$-group; (ii) there exists a subset $\Lambda$ of $D(\Omega)$ such that $\Lambda S \subseteq \Lambda$ and $\Lambda$ generates $\Omega$.

If we wish to specify the distributive subset $\Lambda$ we shall speak of the d.g. right $(R, S)$-group $(\Omega, \Lambda)$.

Definition 2.4. A d.g. near-ring $R$ is said to be a division d.g. near-ring if

(i) $R$ has no non-trivial right ideals;

(ii) $S^{*}=S \backslash\{0\}$ forms a multiplicative group for some distributive semigroup $S$ generating $(R,+)$.

Definition 2.5. A d.g. near-ring $R$ is said to be a regular d.g. near-ring if there exists a distributive semigroup $S$ generating $(R,+)$ and such that

(i) every right ideal of $R$ is a d.g. right $(R, S)$-module;

(ii) for each $t \in S$ there exists $s \in S$ such that $t s t=t$.

Definition 2.6. The centre $C(R)$ of $(R,+)$ is called the additive centre of the d.g. near-ring $R$. Let $Z_{s}=\{s \in S: s t=t s$ for all $t \in S\}$ and $Z_{s}(R)$ be the subgroup of $(R,+)$ generated by $Z_{S}$. $Z_{S}$ and $Z_{S}(R)$ are called the centre of $S$ and the $S$-centre of $R$ respectively.

Proposition 2.7. The $S$-centre of $R$ is a d.g. near-ring and $t z=z t$ for all $z \in Z_{s}(R)$ and $t \in S$.

Proof. By Proposition 2.5 of [17] we have $Z_{s}(R)$ is a d.g. near-ring. Since $z \in Z_{s}(R)$ we have $z=\sum \epsilon_{i} s_{i}$ with $\epsilon_{i}= \pm 1, s_{i} \in Z_{S}$ for all $i$ and consequently $t z=t \sum \epsilon_{i} s_{i}=\sum \epsilon_{i} t s_{i}=\sum \epsilon_{i} s_{i} t=\left(\sum \epsilon_{i} s_{i}\right) t=z t$.

\section{Matrices}

Definition 3.1. An $R$-word $w\left(x_{1}, \ldots, x_{n}\right)$ in the $n$ variables $x_{1}, \ldots, x_{n}$ is a formal expression of the form $\sum a_{i} x_{i}^{\prime}$ with $a_{i} \in R$ and $x_{i}^{\prime} \in\left\{x_{1}, \ldots, x_{n}\right\}$ and $w\left(x_{1}, \ldots, x_{n}\right)$ is said to be a reduced word if the $a_{i}$ are non-zero and $x_{i}^{\prime} \neq x_{i+1}^{\prime}$ for all $i$.

Clearly any word has a unique reduced form and we define the sum of two words 
by juxtaposition and reduction. Further if $w=\sum a_{i} x_{i}^{\prime}$ and $s \in S$ we define $s w=\sum\left(s a_{i}\right) x_{i}^{\prime}$. Thus the set $\Gamma_{n}$ of all reduced $R$-words in the $n$ variables $x_{i}, \ldots, x_{n}$ forms an $S$-group and hence a left $(R, S)$-group.

Now let $\Omega, \Omega^{\prime}$ and $\Omega^{\prime \prime}$ be $v(R,+)$-free left $(R, S)$-groups on $\Lambda=\left\{\lambda_{1}, \ldots, \lambda_{m}\right\}, \Lambda^{\prime}=$ $\left\{\lambda_{1}^{\prime}, \ldots, \lambda_{m}^{\prime}\right\}$ and $\Lambda^{\prime \prime}=\left\{\lambda_{1}^{\prime \prime}, \ldots, \lambda_{m}^{\prime \prime}\right\}$ respectively and let $\phi: \Omega \rightarrow \Omega^{\prime}, \psi: \Omega \rightarrow \Omega^{\prime}$ and $\eta: \Omega^{\prime} \rightarrow \Omega^{\prime \prime}$ be $R$-homomorphisms. We define the sum $\phi+\psi$ of $\phi$ and $\psi$ to be the unique $R$-homomorphism from $\Omega$ to $\Omega^{\prime}$ which maps $\lambda$ onto $\lambda \phi+\lambda \psi$ for all $\lambda \in \Lambda$ and the product $\phi \eta$ as the composition of maps. Thus we have $\lambda(\phi+\psi)=\lambda \phi+\lambda \psi$ and $\lambda(\phi \eta)=(\lambda \phi) \eta$ for all $\lambda \in \Lambda$.

Now the elements of the above groups are expressible, though not uniquely, as $R$-words on their sets of generators and thus we have

$$
\begin{gathered}
\lambda_{i} \phi=A_{i}\left(\lambda_{1}^{\prime}, \ldots, \lambda_{n}^{\prime}\right), \quad \lambda_{i} \psi=B_{i}\left(\lambda_{1}^{\prime}, \ldots, \lambda_{n}^{\prime}\right), \quad \lambda_{j}^{\prime} \eta=C_{j}\left(\lambda_{1}^{\prime \prime}, \ldots, \lambda_{p}^{\prime \prime}\right), \\
\lambda_{i}(\phi+\psi)=D_{i}\left(\lambda_{1}^{\prime}, \ldots, \lambda_{n}^{\prime}\right), \quad \lambda_{i}(\phi \eta)=E_{i}\left(\lambda_{1}^{\prime \prime}, \ldots, \lambda_{p}^{\prime \prime}\right)
\end{gathered}
$$

where the $A_{i}, B_{i}, C_{j}, D_{i}$ and $E_{i}$ are $R$-words on the respective generators. Now $D_{i}\left(\lambda_{1}^{\prime}, \ldots, \lambda_{n}^{\prime}\right)=\lambda_{i}(\phi+\psi)=A_{i}\left(\lambda_{1}^{\prime}, \ldots, \lambda_{n}^{\prime}\right)+B_{i}\left(\lambda_{1}^{\prime}, \ldots, \lambda_{n}^{\prime}\right)$ and $E_{i}\left(\lambda_{1}^{\prime \prime}, \ldots, \lambda_{p}^{\prime \prime}\right)=\lambda_{i}(\phi \eta)=$ $\left(\lambda_{i} \phi\right) \eta=A_{i}\left(\lambda_{1}^{\prime}, \ldots, \lambda_{n}^{\prime}\right) \eta=A_{i}\left(\lambda_{1}^{\prime} \eta, \ldots, \lambda_{n}^{\prime} \eta\right)=A_{i}\left(C_{1}\left(\lambda_{1}^{\prime \prime}, \ldots, \lambda_{p}^{\prime \prime}\right), \ldots, C_{n}\left(\lambda_{1}^{\prime \prime}, \ldots, \lambda_{p}^{\prime \prime}\right)\right)$, and as the $R$-homomorphisms are uniquely determined by these components, we may represent them by these components. For instance, we may represent $\phi$ by a column matrix having $m$ rows with $A_{i}\left(\lambda_{1}^{\prime}, \ldots, \lambda_{n}^{\prime}\right)$ as the element in the $i$ th row. We will use this representation to introduce our matrices over the d.g. near-ring $R$.

In $\Gamma_{n}$ we define $w_{1}\left(x_{1}, \ldots, x_{n}\right)=w_{2}\left(x_{1}, \ldots, x_{n}\right)$ if $w_{1}(\underline{r})=w_{2}(\underline{r})$ for all $\underline{r} \in R^{n}$. Clearly this is an equivalence relation and the equivalence class of the empty word forms a normal $(R, S)$-subgroup $\Delta_{n}$ of $\Gamma_{n}$ and the other equivalence classes are the cosets of this normal subgroup. Further the difference group $\Gamma_{n}-\Delta_{n}$ is a left $(R, S)$-group.

Definition 3.2. A $m \times n$ matrix is a column vector having $m$ rows with an $R$-word in $n$ variables in each of the rows.

For typographical reasons we shall write them in the transposed form with square brackets; for example

$$
A=\left[A_{1}\left(x_{1}, \ldots, x_{n}\right), \ldots, A_{m}\left(x_{1}, \ldots, x_{n}\right)\right]^{\prime}
$$

Two matrices $A$ and $B$ are said to be equal if they are of the same order and their corresponding rows are equivalent.

Let

$$
\begin{aligned}
& A_{m \times n}=\left[A_{1}\left(x_{1}, \ldots, x_{n}\right), \ldots, A_{m}\left(x_{1}, \ldots, x_{n}\right)\right]^{\prime}, \\
& B_{m \times n}=\left[B_{1}\left(x_{1}, \ldots, x_{n}\right), \ldots, B_{m}\left(x_{1}, \ldots, x_{n}\right)\right]^{\prime}, \\
& C_{n \times p}=\left[C_{1}\left(x_{1}, \ldots, x_{p}\right), \ldots, C_{n}\left(x_{1}, \ldots, x_{p}\right)\right]^{\prime}
\end{aligned}
$$


and $r \in R$. We define

$$
\begin{gathered}
A+B=\left[A_{1}\left(x_{1}, \ldots, x_{n}\right)+B_{1}\left(x_{1}, \ldots, x_{n}\right), \ldots, A_{m}\left(x_{1}, \ldots, x_{n}\right)+B_{m}\left(x_{1}, \ldots, x_{n}\right)\right]^{\prime} \\
r A=\left[r A_{1}\left(x_{1}, \ldots, x_{n}\right), \ldots, r A_{m}\left(x_{1}, \ldots, x_{n}\right)\right]^{\prime}
\end{gathered}
$$

and

$$
A C=D=\left[D_{1}\left(x_{1}, \ldots, x_{p}\right), \ldots, D_{m}\left(x_{1}, \ldots, x_{p}\right)\right]^{\prime}
$$

where

$$
D_{i}\left(x_{1}, \ldots, x_{p}\right)=A_{i}\left(C_{1}\left(x_{1}, \ldots, x_{p}\right), \ldots, C_{n}\left(x_{1}, \ldots, x_{p}\right)\right)
$$

Let $I_{n \times n}=\left\{x_{1}, \ldots, x_{n}\right]^{\prime}$ and $O_{m \times n}=[0, \ldots, 0]^{\prime}$. We then have

Proposition 3.3. (i) $I_{m \times m} A_{m \times n}=A_{m \times n}$;

(ii) $A_{m \times n} I_{n \times n}=A_{m \times n}$;

(iii) $O_{m \times n} A_{n \times p}=O_{m \times p}$;

(iv) $A_{m \times n} O_{n \times p}=O_{m \times p}$;

(v) $A_{m \times n}+O_{m \times n}=A_{m \times n}=O_{m \times n}+A_{m \times n}$.

$I_{n \times n}$ will be called the $n \times n$ identity matrix and $O_{m \times n}$ the zero $m \times n$ matrix.

Definition 3.4. A matrix $A$ is said to be

(i) a scalar matrix if it is of the form $r I$ with $r \in R$;

(ii) a diagonal matrix if the coefficients of $x_{j}$ in $A_{i}\left(x_{1}, \ldots, x_{n}\right)$ are zero for all $j \neq i$;

(iii) upper triangular if the coefficients of $x_{j}$ in $A_{i}\left(x_{1}, \ldots, x_{n}\right)$ are zero for all $j<i$;

(iv) strictly upper triangular if the coefficients of $x_{j}$ in $A_{i}\left(x_{1}, \ldots, x_{n}\right)$ are zero for all $j \leq i$

(v) lower triangular if the coefficients of $x_{j}$ in $A_{i}\left(x_{1}, \ldots, x_{n}\right)$ are zero for all $j>i$; $j \geq i$.

(vi) strictly lower triangular if the coefficients of $x_{j}$ in $A_{i}\left(x_{1}, \ldots, x_{n}\right)$ are zero for all

If $A$ is an $m \times n$ matrix and $T_{i j}, T_{i+r j}$ and $T_{r j}$ are the elementary row matrices obtained from the $m \times m$ identity matrix $I$ by interchanging the $i$ th and $j$ th rows, adding $r$ times the $j$ th row to the $i$ th row of $I$ and multiplying the $j$ th row of $I$ by $r$ respectively (where $r$ is an element of $R$ ), then it can be easily verified that $T_{i j} A, T_{i+r j} A$ and $T_{r j} A$ are the matrices obtained from $A$ by performing the corresponding row operations on $A$. 


\section{Matrix d.g. near-ring}

We will denote by $M_{n}(R)$ the set of all $n \times n$ matrices over the d.g. near-ring $R$ and define $M_{n}(S)=\left\{A \in M_{n}(R): A_{i}\left(x_{1}, \ldots, x_{n}\right)=s_{i} x_{i}^{\prime}\right.$ with $s_{i} \in S$ and $x_{i}^{\prime} \in\left\{x_{1}, \ldots, x_{n}\right\}$ for all $1 \leq i \leq n\}$.

Then it can be easily verified that

(i) $M_{n}(R)$ forms a right near-ring with identity $I$;

(ii) $M_{n}(S)$ forms a distributive semigroup in $M_{n}(R)$;

(iii) $M_{n}(S)$ generates $\left(M_{n}(R),+\right)$;

and consequently $M_{n}(R)$ is a d.g. near-ring.

For the rest of this paper we will assume that $\Omega$ is a $v(R,+)$-free left $(R, S)$-group on $\Lambda=\left\{\lambda_{1}, \ldots, \lambda_{n}\right\}$ and $\bar{R}$ denotes the endomorphism d.g. near-ring of $\Omega$ (cf. Proposition 2.2 of [18]). Also let $\bar{S}=\{\bar{x} \in \bar{R}: \Lambda \bar{x} \subseteq S \Lambda\}$.

Theorem 4.1. $\left(M_{n}(R), M_{n}(S)\right)$ is d.g. near-ring isomorphic to $(\bar{R}, \bar{S})$.

Proof. Let $\phi: M_{n}(R) \rightarrow \bar{R}$ be defined by $\lambda_{i} \phi(A)=A_{i}\left(\lambda_{1}, \ldots, \lambda_{n}\right)$. Since $\Omega$ is a $v(R,+)$-free left $(R, S)$-group, we have $A_{i}\left(x_{1}, \ldots, x_{n}\right)=0$ if and only if $A_{i}\left(\lambda_{1}, \ldots, \lambda_{n}\right)=0$ and consequently $\phi$ is well defined, as an $R$-endomorphism of $\Omega$ is uniquely determined by its action on $\Lambda$. It can be easily verified that $\phi$ is a near-ring epimorphism. Now suppose $\phi(A)=0$. Then $A_{i}\left(\lambda_{1}, \ldots, \lambda_{n}\right)=0$ for $i=1, \ldots, n$ and since $\Omega$ is $v(R,+)$-free we have $A_{i}(\underline{r})=0$ for all $\underline{r} \in R^{n}$. Consequently $A_{i}\left(x_{1}, \ldots, x_{n}\right)=0$ for $i=1, \ldots, n$ and thus $\phi$ is a near-ring isomorphism. Clearly $\phi\left(M_{n}(S)\right)=\bar{S}$.

Proposition 4.2. If $R$ is a ring with identity and $S=R$, then $\Omega$ is a free $R$-module on $\Lambda=\left\{\lambda_{1}, \ldots, \lambda_{n}\right\}$

Proof. Since $\Omega$ is $v(R,+)$-free and $(R,+)$ is abelian we have $\Omega$ to be abelian and consequently $\Omega$ is an $R$-module. Thus any element of $\Omega$ can be represented in the form $\sum_{i=1}^{n} a_{i} \lambda_{i}$ and as $\Omega$ is a $v(R,+)$-free left $(R, S)$-group we have $\sum_{i=1}^{n} a_{i} \lambda_{i}=0$ if and only if $\sum_{i=1}^{n} a_{i} r_{i}=0$ for all $r_{1}, \ldots, r_{n}$ in $R$.

Hence $\sum_{i=1}^{n} a_{i} \lambda_{i}=0$ if and only if $a_{i}=0$ for all $i$ and consequently $\Omega$ is a free $R$ module on $\Lambda$.

Thus our matrix d.g. near-rings reduce to ordinary matrix rings when $R$ is a ring.

Now let $\phi_{0}: R \rightarrow \bar{R}$ be defined by $\lambda \phi_{0}(x)=x \lambda$. for all $\lambda \in \Lambda$ and $x \in R$. Then, as $R \in v(R,+)$, by Proposition 2.5 of [18] we have, by identifying $R$ with $\phi_{0}(R)$,

Proposition 4.3. (i) $R$ is a sub near-ring of $\bar{R}$;

(ii) $R$ is a sub near-ring of $M_{n}(R)$. 
Now let $E_{i j}$ denote the $n \times n$ matrix having $x_{j}$ as the element in the ith row and zero elsewhere and let $\bar{e}_{\lambda_{i} \lambda_{j}}$ be the element of $\bar{R}$ which maps $\lambda_{i}$ onto $\lambda_{j}$ and $\lambda_{\text {onto }} 0$ for all $\lambda\left(\neq \lambda_{i i}\right)$ in $\Lambda$. We will denote $\bar{e}_{\lambda_{i, \lambda_{i}}}$ by $\bar{e}_{\lambda_{i}}$.

Theorem 4.4. (i) $\bar{R}$ is a $v(R,+)$-left $(R, S)$-group on $\left\{\bar{e}_{\lambda_{i} \lambda_{j}}: 1 \leq i, j \leq n\right\}$;

(ii) $M_{n}(R)$ is a $v(R,+)$-left $(R, S)$-group on $\left\{E_{i j}: 1 \leq i, j \leq n\right\}$.

Proof. (i) If $\bar{x} \in \bar{R}, \lambda \in \Lambda$ and $\lambda \bar{x}=\sum a_{i} \lambda_{i}^{\prime}$ with $a_{i} \in R$ and $\lambda_{i}^{\prime} \in \Lambda$, we have $\bar{e}_{j}, \bar{x}=\sum a_{i} \bar{e}_{\lambda_{i, j}^{\prime}}$ and consequently $\left\{\bar{e}_{\lambda_{i,} \lambda_{j}}: 1 \leq i, j \leq n\right\}$ generates $\bar{R}$ as $\bar{x}=\sum_{i=1}^{n} \bar{e}_{\lambda_{i}} \bar{x}$. By Theorem 3, Corollary 4 of [18] we have $v(R,+)=v(\bar{R},+)$ and so $\bar{R}$ is a $v(R,+)$-left $(R, S)$-group.

(ii) This follows from (i) and Theorem 4.1.

Proposition 4.5. (i) $\left\{E_{i i}: 1 \leq i \leq n\right\}$ is an orthogonal set of idempotents in $M_{n}(R)$;

(ii) The set of all diagonal matrices in $M_{n}(R)$ is a sub near-ring which is near-ring isomorphic to $R^{n}$;

(iii) The set of all upper triangular (lower triangular, strictly upper triangular, strictly lower triangular) matrices in $M_{n}(R)$ is a sub d.g. near-ring;

(iv) The set of all strictly upper triangular (lower triangular) matrices in $M_{n}(R)$ is an ideal in the d.g. near-ring of upper triangular (lower triangular) matrices.

The proof of this Proposition is straightforward and will be omitted.

Definition 4.6. An $m \times n$ matrix $A$ is said to be

(i) a kth column matrix if $A_{i}\left(x_{1}, \ldots, x_{n}\right)=a_{i} x_{k}$ for all $i$;

(ii) a kth row matrix if $A_{i}\left(x_{1}, \ldots, x_{n}\right)=0$ for all $i \neq k$.

Clearly we have (i) any matrix is a sum of column matrices; (ii) any matrix is a sum of row matrices; and (iii) the matrices $E_{i j}$ are column as well as row matrices.

It can be easily verified that the set of all $k$ th column matrices in $M_{n}(R)$ is a left $M_{n}(R)$ submodule and the set of all $k$ th row matrices is a right ideal in $M_{n}(R)$.

Now by Propositions 5.3 and 5.4 of [18] and Theorem 4.1 we have

Theorem 4.7. (i) $R$ is left primitive if and only if $M_{n}(R)$ is left primitive;

(ii) $R$ is simple if and only if $M_{n}(R)$ is simple.

By Theorem 6 of [20] and Theorem 4.1 we have the following generalisation of the Wedderburn-Artin theorem.

Theorem 4.8. If $R$ is a discrete d.g. near-ring satisfying the descending chain condition for right ideals then the following three conditions are equivalent: 
(i) $R$ is simple and has an irreducible d.g. right $(R, S)$-module for some $S$;

(ii) $R$ is right primitive;

(iii) $R$ is near-ring isomorphic to $M_{n}\left(R_{1}\right)$ for some division d.g. near-ring $R_{1}$ and some positive integer $n$.

By Theorems 6 and 7 of [21] and Theorem 4.1 we have

Theorem 4.9. (i) If $(R, S)$ is a division d.g. near-ring then $\left(M_{n}(R), M_{n}(S)\right)$ is a regular d.g. near-ring;

(ii) If $(R, D(R))$ is a division d.g. near-ring then $\left(M_{n}(R), D\left(M_{n}(R)\right)\right)$ is a regular d.g. near-ring.

Proposition 4.10. $R$ is near-ring isomorphic to $\bar{e}_{\lambda}, \bar{R} \bar{e}_{\lambda}$.

Proof. Define $\phi: R \rightarrow \bar{e}_{\lambda} \bar{R} \bar{e}_{\lambda}$ by $\lambda \phi(x)=x \lambda$ and $\lambda^{\prime} \phi(x)=0$ if $\lambda^{\prime} \neq \lambda$. It can be easily verified that $\phi$ is a near-ring homomorphism. Now given $\bar{e}_{\lambda} \overline{x e}_{\lambda} \in \bar{e}_{\lambda} \bar{R}_{\bar{e}_{\lambda}}$ we have $\lambda \bar{e}_{\lambda} \overline{x e}_{\lambda}=x \lambda$ for some $x \in R$ and $\lambda^{\prime} \bar{e}_{\lambda} \overline{x e}_{\lambda}=0$ for all $\lambda^{\prime} \neq \lambda$. Thus $\phi(x)=\bar{e}_{\lambda} \overline{x e}_{\lambda}$ and so $\phi$ is onto. Now suppose $\phi(x)=0$. Then $x \lambda=0$ and as $\Omega$ is a $v(R,+)$-free group we have $x y=0$ for all $y \in R$ and in particular $x=x e=0$. Thus $\phi$ is an isomorphism.

Proposition 4.11. $\bar{R} \cdot \bar{e}_{\lambda} \cdot \bar{R}=\bar{R}$ where $\bar{R} \cdot \bar{e}_{\lambda} \cdot \bar{R}=\left\{\sum \bar{x}_{i} \bar{e}_{i} \bar{y}_{i}: \bar{x}_{i}, \bar{y}_{i} \in \bar{R}\right\}$.

Proof. Clearly we have $\bar{R} \cdot \bar{e}_{\lambda} \cdot \bar{R} \subseteq \bar{R}$ and let $\bar{x} \in \bar{R}$. Then $\lambda_{i} \bar{x}=\sum_{j=1}^{k_{i}} a_{i j} \lambda_{i j}^{\prime}=$ $\lambda_{i} \sum_{j=1}^{k_{i}} a_{i j} \bar{e}_{\lambda_{i} \lambda_{i j}^{\prime}}=\lambda_{i} \sum_{j=1}^{k_{i}} a_{i j} \bar{e}_{\lambda_{i}} \bar{e}_{\lambda}, \bar{e}_{\lambda \lambda_{i j}^{\prime}}$ and thus if $\bar{y}_{i}=\sum_{j=1}^{k_{i}} a_{i j} \bar{e}_{\lambda_{j},} \bar{e}_{\lambda} \bar{e}_{\lambda \lambda_{i j}^{\prime}}$ we have $\bar{y}_{i} \in \bar{R} \cdot \bar{e}_{\lambda} \cdot \bar{R}, \lambda_{i}\left(\bar{x}-\bar{y}_{i}\right)=0$ and $\lambda \bar{y}_{i}=0$ if $\lambda \neq \lambda_{i}$. Define $\bar{y}=\sum_{i=1}^{n} \bar{y}_{i}$. Then $\bar{y} \in \bar{R} \cdot \bar{e}_{\lambda} \cdot \bar{R}$ and $\lambda_{i}(\bar{x}-\bar{y})=0$ for all $i$. Hence $\bar{x}=\bar{y} \in \bar{R} \cdot \bar{e}_{\lambda} \cdot \bar{R}$ and the result follows.

Combining Propositions 4.10 and 4.11 we have

Theorem 4.12. There exists an idempotent $E$ in $M_{n}(R)$ such that $E M_{n}(R) E \cong R$ and $M_{n}(R) \cdot E \cdot M_{n}(R)=M_{n}(R)$.

We note that two rings $R$ and $S$ are Morita equivalent if and only if there exists an idempotent $e$ in $S$ such that

$$
e S e \cong R \text { and } \quad \text { S.e.S }=S
$$

and thus Theorem 4.12 is a generalisation of the result that $R$ and $M_{n}(R)$ are Morita equivalent when $R$ is a ring.

Theorem 4.13. $Z_{\bar{s}}(\bar{R})=\left\{\bar{x} \in \bar{R}: \bar{x}=r \bar{e}\right.$ with $\left.r \in Z_{s}(R)\right\}$ where $\bar{e}$ is the identity of $\bar{R}$. 
Proof. Let $\bar{x} \in Z_{\bar{s}}(\bar{R})$. Then $\overline{x e}_{\lambda_{i, \lambda_{j}}}=\bar{e}_{\lambda_{i} \lambda_{j}} \bar{x}$ for all $i, j$ and so $\lambda_{i} \overline{x e}_{\lambda_{i} \lambda_{j}}=\lambda_{j j} \bar{x}$ for all $i, j$. Consequently we have $\lambda_{i} \bar{x}=r \lambda_{i}$ for all $i$ and thus $\bar{x}=r \bar{e}$ with $r \in R$. Thus if $\bar{t} \in Z_{\bar{s}}$ we have $\bar{t}=t \bar{e}$ with $t \in S$ and, using $\bar{t}(s \bar{e})=(s \bar{e}) \bar{t}$ with $s \in S$, we get $t \in Z_{s}$. Hence $\bar{x}=\sum \epsilon_{i} \bar{t}_{i}=\sum \epsilon_{i}\left(t_{i} \bar{e}\right)=\sum\left(\epsilon_{i} t_{i}\right) \bar{e}=r \bar{e}$ with $r \in Z_{S}(R)$ and $\epsilon_{i}= \pm 1$. Conversely let $r \in Z_{S}(R)$. Then $r=\sum \epsilon_{i} t_{i}$ with $t_{i} \in Z_{S}$ and so $\left(t_{i} \bar{e}\right) \bar{t}=\bar{t}\left(t_{i} \bar{e}\right)$ for all $\bar{t} \in \bar{S}$. Thus $t_{i} \bar{e} \in Z_{\bar{S}}$ and consequently $r \bar{e} \in Z_{\bar{s}}(\bar{R})$.

Corollary 4.14. $Z_{\bar{s}}(\bar{R}) \cong Z_{S}(R)$.

This corollary is a generalisation of the result that the centre of a matrix ring is ring isomorphic to the centre of the base ring.

\section{Dual $(R, S)$-groups}

Let $\Omega^{*}=\operatorname{Hom}_{R}(\Omega, R)$ be the set of all left $R$-homomorphisms from $\Omega$ into $R$. If $x \in R$ and $\alpha^{*}, \beta^{*} \in \Omega^{*}$, denote by $\alpha^{*}+\beta^{*}$ and $\alpha^{*} x$ the unique $R$-homomorphisms from $\Omega$ to $R$ defined respectively by $\lambda\left(\alpha^{*}+\beta^{*}\right)=\lambda \alpha^{*}+\lambda \beta^{*}$ and $\lambda\left(\alpha^{*} x\right)=\left(\lambda \alpha^{*}\right) x$ for all $\lambda \in \Lambda$. For each $\lambda \in \Lambda$ let $\lambda^{*}$ denote the element of $\Omega^{*}$ defined by $\lambda \lambda^{*}=e$ and $\lambda^{\prime} \lambda^{*}=0$ for all $\lambda^{\prime}(\neq \lambda)$ in $\Lambda$. Also let $\Lambda_{s}^{*}=\left\{\alpha^{*} \in \Omega^{*}: \lambda \alpha^{*} \in S\right.$ for all $\left.\lambda \in \Lambda\right\}$ and $\Lambda^{*}=\left\{\lambda^{*}: \lambda \in \Lambda\right\}$.

Proposition 5.1. $\left(\Omega^{*}, \Lambda_{S}^{*}\right)$ is a d.g. right $(R, S)$-group.

Proof. Let $x, y \in R$ and $s \in S$. We have $\lambda\left(\left(\alpha^{*}+\beta^{*}\right) x\right)=\left(\lambda \alpha^{*}+\lambda \beta^{*}\right) x=\left(\lambda \alpha^{*}\right) x+$ $\left(\lambda \beta^{*}\right) x=\lambda\left(\alpha^{*} x\right)+\lambda\left(\beta^{*} x\right), \quad \lambda\left(\alpha^{*}(x y)\right)=\left(\lambda \alpha^{*}\right)(x y)=\left(\left(\lambda \alpha^{*}\right) x\right) y=\left(\lambda\left(\alpha^{*} x\right)\right) y=\lambda\left(\left(\alpha^{*} x\right) y\right) \quad$ and $\lambda\left(\alpha^{*} e\right)=\left(\lambda \alpha^{*}\right) e=\lambda \alpha^{*}$ for all $\lambda \in \Lambda$ and thus $\Omega^{*}$ is a right $R$-group. Also if $\alpha^{*} \in \Lambda_{s}^{*}$ we have $\lambda\left(\alpha^{*}(x+y)\right)=\left(\lambda \alpha^{*}\right)(x+y)=\left(\lambda \alpha^{*}\right) x+\left(\lambda \alpha^{*}\right) y=\lambda\left(\alpha^{*} x\right)+\lambda\left(\alpha^{*} y\right)$ and $\lambda\left(\alpha^{*} s\right)=\left(\lambda \alpha^{*}\right) s \in S$ for all $\lambda \in \Lambda$.

Thus $\Lambda_{s}^{*}$ is a set of distributive elements in $\Omega^{*}$ such that $\Lambda_{s}^{*} S \subseteq \Lambda_{s}^{*}$. Now given $\alpha^{*} \in \Omega^{*}$ let $\lambda_{i} \alpha^{*}=\sum_{j=1}^{k_{i}} \epsilon_{i j} s_{i j}$ with $\epsilon_{i j}= \pm 1$ and $s_{i j} \in S$. Let $\gamma_{i j}^{*}$ be the element of $\Omega^{*}$ defined by $\lambda_{i} \gamma_{i j}^{*}=s_{i j}$ and $\lambda \gamma_{i j}^{*}=0$ for all $\lambda\left(\neq \lambda_{i}\right)$ in $\Lambda$. Then $\gamma_{i j}^{*} \in \Lambda_{s}^{*}$ and $\alpha^{*}=\sum_{i=1}^{n} \sum_{j=1}^{k_{i}} \epsilon_{i j} \gamma_{i j}^{*}$. Thus $\Lambda_{s}^{*}$ generates $\Omega^{*}$ and the result follows.

Proposition 5.2. $\omega\left(\omega^{*} x\right)=\left(\omega \omega^{*}\right) x$ for all $\omega \in \Omega, \omega^{*} \in \Omega^{*}$ and $x \in R$.

Proof. If $\omega=\sum \pm s_{i} \dot{\lambda}_{i}^{\prime}$ with $s_{i} \in S$ and $\lambda_{i} \in \Lambda$ we have $\omega\left(\omega^{*} x\right)=\left(\sum \pm s_{i} \lambda_{i}^{\prime}\right)\left(\omega^{*} x\right)=$ $\sum \pm\left(s_{i} \lambda_{i}^{\prime}\right)\left(\omega^{*} x\right)=\sum \pm s_{i}\left(\lambda_{i}^{\prime}\left(\omega^{*} x\right)\right)=\sum \pm s_{i}\left(\lambda_{i}^{\prime} \omega^{*}\right) x=\left(\sum \pm s_{i}\left(\lambda_{i}^{\prime} \omega^{*}\right)\right) x=\left(\left(\sum \pm s_{i} \lambda_{i}^{\prime}\right) \omega^{*}\right) x=\left(\omega \omega^{*}\right) x$

Now by Proposition 3.2 of [18] and Proposition 4.3 we have

Proposition 5.3. (i) $\Omega^{*}$ is a left $(\bar{R}, \bar{S})$-group with $\bar{x} \alpha^{*}$ being defined by $\lambda\left(\bar{x} \alpha^{*}\right)=(\lambda \bar{x}) \alpha^{*}$ for all $\lambda \in \Lambda$;

(ii) $\Omega^{*}$ is a left $(R, S)$-group. 
By the Corollary 3 of Theorem 3 of [18] we have

Proposition 5.4. $\Omega^{*} \in v(R,+)$.

Now given $x \in R, \bar{x} \in \bar{R}$ and $\alpha^{*} \in \Omega^{*}$, we have $\lambda\left(\left(\bar{x} \alpha^{*}\right) x\right)=\left(\lambda\left(\bar{x} \alpha^{*}\right)\right) x=\left((\lambda \bar{x}) \alpha^{*}\right) x=$ $(\lambda \bar{x})\left(\alpha^{*} x\right)=\lambda\left(\bar{x}\left(\alpha^{*} x\right)\right)$ for all $\lambda \in \Lambda$ and consequently we have

Proposition 5.5. $\left(\bar{x} \alpha^{*}\right) x=\bar{x}\left(\alpha^{*} x\right)$ for all $x \in R, \bar{x} \in \bar{R}$ and $\alpha^{*} \in \Omega^{*}$.

Theorem 5.6. (i) $\left(\Omega^{*}, \Lambda_{S}^{*}\right)$ is right $R$-isomorphic to $\left(R^{n}, S^{n}\right)$;

(ii) An element of $\Omega^{*}$ has a unique representation in the form $\sum_{i=1}^{n} \lambda_{i}^{*} x_{i}$ with $x_{i} \in R$;

(iii) $\Lambda^{*}$ generates $\Omega^{*}$ as a right $R$-group and $\Lambda^{*} \subseteq \Lambda_{s}^{*}$.

Proof. (i) Define $\phi: \Omega^{*} \rightarrow R^{n}$ by $\left(\phi\left(\alpha^{*}\right)\right)_{i}=\lambda_{i} \alpha^{*}$ for $i=1, \ldots, n$. Then from the proofs of Theorems 2 and 3 of [18] we have $\phi$ is a left $R$-isomorphism. Now $\left(\phi\left(\alpha^{*} x\right)\right)_{i}=\lambda_{i}\left(\alpha^{*} x\right)=\left(\lambda_{i} \alpha^{*}\right) x=\left(\phi\left(\alpha^{*}\right)\right)_{i} x$ for $i=1, \ldots, n$ and so $\phi$ is a right $R$ homomorphism. Also clearly we have $\phi\left(\Lambda_{s}^{*}\right)=S^{n}$.

(ii) Since $\Omega^{*}$ is right $R$-isomorphic to $R^{n}$ and $\left(\phi\left(\lambda_{j}^{*}\right)\right)_{i}=e$ if $j=i$ and is zero if $j \neq i$, any element of $\Omega^{*}$ has a unique representation in the form $\sum_{i=1}^{n} \lambda_{i}^{*} x_{i}$.

(iii) This follows from (ii) and the definition of $\Lambda_{s}^{*}$.

Definition 5.7. The d.g. right $(R, S)$-group $\Omega^{*}$ is called the dual of the left $(R, S)$ group $\Omega$ and $\Lambda^{*}$ the dual basis to $\Lambda$.

Now let $\operatorname{hom}_{R}^{r}\left(\Omega^{*}, R\right)$ be the set of all right $R$-homomorphisms from $\Omega^{*}$ to $R$ and let $\Omega^{* *}=\operatorname{Hom}_{R}\left(\Omega^{*}, R\right)$ where $\operatorname{Hom}_{R}\left(\Omega^{*}, R\right)$ is the subgroup of $\operatorname{Map}\left(\Omega^{*}, R\right)$ generated by $\operatorname{hom}_{R}^{r}\left(\Omega^{*}, R\right)$.

Given $x \in R$ and $\omega^{* *} \in \Omega^{* *}$ define $x \omega^{* *}$ by $\left(x \omega^{* *}\right) \omega^{*}=x\left(\omega^{* *} \omega^{*}\right)$ for all $\omega^{*} \in \Omega^{*}$.

Proposition 5.8. (i) $S . h_{R}^{\prime}\left(\Omega^{*}, R\right) \subseteq$ hom $_{R}^{r}\left(\Omega^{*}, R\right)$;

(ii) $\Omega^{* *}$ is a left $(R, S)$-group.

Proof. (i) Let $s \in S$ and $\omega^{* *} \in$ hom $m_{R}^{r}\left(\Omega^{*}, R\right)$. Then $\left(s \omega^{* *}\right)\left(\omega_{1}^{*}+\omega_{2}^{*}\right)=s\left(\omega^{* *}\left(\omega_{1}^{*}+\omega_{2}^{*}\right)\right)=$ $s\left(\omega^{* *} \omega_{1}^{*}+\omega^{* *} \omega_{2}^{*}\right)=s\left(\omega^{* *} \omega_{1}^{*}\right)+s\left(\omega^{* *} \omega_{2}^{*}\right)=\left(s \omega^{* *}\right) \omega_{1}^{*}+\left(s \omega^{* *}\right) \omega_{2}^{*}$ for all $\omega_{1}^{*}, \omega_{2}^{*} \in \Omega^{*}$, and $\left(s \omega^{* *}\right)\left(\omega^{*} x\right)=s\left(\omega^{* *}\left(\omega^{*} x\right)\right)=s \cdot\left(\left(\omega^{* *} \omega^{*}\right) x\right)=\left(s \cdot\left(\omega^{* *} \omega^{*}\right)\right) x=\left(\left(s \omega^{* *}\right) \omega^{*}\right) x$ for all $\omega^{*} \in \Omega^{*}$ and $x \in R$. Thus $s \omega^{* *} \in \operatorname{hom}_{R}^{r}\left(\Omega^{*}, R\right)$.

(ii) By (i) we have $x \omega^{* *} \in \Omega^{* *}$ for all $x \in R$ and $\omega^{* *} \in \Omega^{* *}$. Clearly $e \omega^{* *}=\omega^{* *}$. Now given $s \in S$ and $\omega_{1}^{* *}, \omega_{2}^{* *} \in \Omega^{* *}$ we have $\left(s\left(\omega_{1}^{* *}+\omega_{2}^{* *}\right)\right)\left(\omega^{*}\right)=s\left(\left(\omega_{1}^{* *}+\omega_{2}^{* *}\right) \omega^{*}\right)=$ $s\left(\omega_{1}^{* *} \omega^{*}+\omega_{2}^{* *} \omega^{*}\right)=s\left(\omega_{1}^{* *} \omega^{*}\right)+s\left(\omega_{2}^{* *} \omega^{*}\right)=\left(s \omega_{1}^{* *}\right) \omega^{*}+\left(s \omega_{2}^{* *}\right) \omega^{*}=\left(s \omega_{1}^{* *}+s \omega_{2}^{* *}\right) \omega^{*}$ and $\left((s t) \omega_{1}^{* *}\right) \omega^{*}=(s t)\left(\omega_{1}^{* *} \omega^{*}\right)=s\left(t\left(\omega_{1}^{* *} \omega^{*}\right)\right)=s\left(\left(t \omega_{1}^{* *}\right) \omega^{*}\right)=\left(s\left(t \omega_{1}^{* *}\right)\right) \omega^{*}$ for all $\omega^{*} \in \Omega^{*}$. Hence $\Omega^{* *}$ is a left $S$-group and consequently is a left $(R, S)$-group. 
Now as, by Theorem 5.6, every element of $\Omega^{*}$ has a unique representation in the form $\sum_{i=1}^{n} \lambda_{i}^{*} x_{i}$, we will for each $i \in\{1, \ldots, n\}$ define a map $\lambda_{i}^{* *}$ from $\Omega^{*}$ to $R$ by $\lambda_{i}^{* *}\left(\sum_{j=1}^{n} \lambda_{j}^{*} x_{j}\right)=x_{i}$ and let $\Lambda^{* *}=\left\{\lambda_{1}^{* *}, \ldots, \lambda_{n}^{* *}\right\}$.

Proposition 5.9. (i) $\Lambda^{* *} \subseteq h_{R}^{\prime}\left(\Omega^{*}, R\right)$;

(ii) $\Lambda^{* *}$ generates $\Omega^{* *}$ as a left $(R, S)$-group.

Proof. (i) $\lambda_{i}^{* *}\left(\sum_{j=1}^{n} \lambda_{j}^{*} x_{j}+\sum_{j=1}^{n} \lambda_{j}^{*} y_{j}\right)=\lambda_{i}^{* *}\left(\sum_{j=1}^{n} \lambda_{j}^{*}\left(x_{j}+y_{j}\right)\right)=x_{i}+y_{i}=\lambda_{i}^{* *}\left(\sum_{j=1}^{n} \lambda_{j}^{*} x_{j}\right)+$ $\lambda_{i}^{* *}\left(\sum_{j=1}^{n} \lambda_{j}^{*} y_{i}\right)$ and $\lambda_{i}^{* *}\left(\left(\sum_{j=1}^{n} \lambda_{j}^{*} x_{j}\right) x\right)=\lambda_{i}^{* *}\left(\sum_{j=1}^{n} \lambda_{j}^{*}\left(x_{j} x\right)\right)=x_{i} x=\left(\lambda_{i}^{* *}\left(\sum_{j=1}^{n} \lambda_{j}^{*} x_{j}\right)\right) x$. Hence $\lambda_{i}^{* *}$ is a right $R$-homomorphism.

(ii) If $\omega^{* *} \in h o m_{R}^{r}\left(\Omega^{*}, R\right)$ and $\omega^{*} \in \Omega^{*}$ then $\omega^{* *}\left(\omega^{*}\right)=\omega^{* *}\left(\sum_{j=1}^{n} \lambda_{j}^{*} x_{j}\right)=\sum_{j=1}^{n}\left(\omega^{* *} \lambda_{j}^{*}\right) x_{j}=$ $\sum_{j=1}^{n}\left(\omega^{* *} \lambda_{j}^{*}\right)\left(\lambda_{j}^{* *}\left(\sum_{i=1}^{n} \lambda_{i}^{*} x_{i}\right)\right)=\left(\sum_{j=1}^{n}\left(\omega^{* *} \lambda_{j}^{*}\right) \lambda_{j}^{* *}\right)\left(\omega^{*}\right)$ for all $\omega^{*} \in \Omega^{*}$ and consequently $\omega^{* *}=\sum_{j=1}^{n}\left(\omega^{* *} \lambda_{j}^{*}\right) \lambda_{j}^{* *}$. But hom ${ }_{R}^{r}\left(\Omega^{*}, R\right)$ generates $\Omega^{* *}$ and thus $\Lambda^{* *}$ generates $\Omega^{* *}$ as a left $(R, S)$-group.

Theorem 5.10. (i) $\Omega^{* *} \in v(R,+)$;

(ii) $\Omega^{* *}$ is left $R$-isomorphic to $\Omega$.

Proof. (i) Let $w\left(x_{1}, \ldots, x_{m}\right)$ be an $R$-word in the $m$ variables $x_{1}, \ldots, x_{m}$ such that $w\left(r_{1}, \ldots, r_{m}\right)=0$ for all $r_{1}, \ldots, r_{m}$ in $R$. Then given $\omega_{1}^{* *}, \ldots, \omega_{m}^{* *} \in \Omega^{* *}$ we have $w\left(\omega_{1}^{* *}, \ldots, \omega_{m}^{* *}\right)\left(\omega^{*}\right)=w\left(\omega_{1}^{* *} \omega^{*}, \ldots, \omega_{m}^{* *} \omega^{*}\right)=0$ for all $\omega^{*} \in \Omega^{*}$ and so $w\left(\omega_{1}^{* *}, \ldots, \omega_{m}^{* *}\right)=0$. Thus $\Omega^{* *} \in v(R,+)$.

(ii) Since $\Omega$ is a $v(R,+)$-free left $(R, S)$-group on $\Lambda$ and $\Omega^{* *} \in v(R,+)$ let $\phi: \Omega \rightarrow \Omega^{* *}$ be the left $R$-homomorphism defined by $\phi\left(\lambda_{i}\right)=\lambda_{i}^{* *}$ for $i=1, \ldots, n$. Now $\phi$ is surjective as $\Lambda^{* *}$ generates $\Omega^{* *}$. Suppose $\phi(\omega)=0$ and $\omega=w\left(\lambda_{1}, \ldots, \lambda_{n}\right)$ where $w$ is an $R$-word. Then $\phi(w)=w\left(\lambda_{1}^{* *}, \ldots, \lambda_{n}^{* *}\right)$ and so $w\left(\lambda_{1}^{* *} \omega^{*}, \ldots, \lambda_{n}^{* *} \omega^{*}\right)=w\left(\lambda_{1}^{* *}, \ldots, \lambda_{n}^{* *}\right)\left(\omega^{*}\right)=0$ for all $\omega^{*} \in \Omega^{*}$.

Now given $r_{1}, \ldots, r_{n}$ in $R$, choosing $\omega^{*}=\sum_{j=1}^{n} \lambda_{j}^{*} r_{j}$ we have $w\left(r_{1}, \ldots, r_{n}\right)=$ $w\left(\lambda_{1}^{* *} \omega^{*}, \ldots, \lambda_{n}^{* *} \omega^{*}\right)=0$ and consequently we have $\omega=0$. Thus $\Omega$ is left $R$-isomorphic to $\Omega^{* *}$.

Definition 5.11. The left $(R, S)$-group $\Omega^{* *}$ is called the dual of the d.g. right $(R, S)$ group $\Omega^{*}$ and the double dual of the left $(R, S)$-group $\Omega$ and $\Lambda^{* *}$ is called the dual basis to $\Lambda^{*}$ and the double dual basis to $\Lambda$.

Definition 5.12. (i) For $\omega \in \Omega$ and $\omega^{*} \in \Omega^{*}$ we denote by $\omega^{*} \omega$ the $R$-endomorphism of $\Omega$ defined by $\lambda\left(\omega^{*} \omega\right)=\left(\lambda \omega^{*}\right) \omega$ for all $\lambda \in \Lambda$.

(ii) $\Omega . \Omega^{*}$ and $\Omega \circ \Omega^{*}$ denote respectively the subgroup and normal subgroup of $(R,+)$ generated by the set $\left\{\omega \omega^{*}: \omega \in \Omega, \omega^{*} \in \Omega^{*}\right\}$ and $\Omega^{*} . \Omega$ and $\Omega^{*} \circ \Omega$ denote respectively the subgroup and normal subgroup of $(\bar{R},+)$ generated by the set $\left\{\omega^{*} \omega: \omega^{*} \in \Omega^{*}, \omega \in \Omega\right\}$. 
Proposition 5.13. (i) $\omega^{*}(x \omega)=\left(\omega^{*} x\right) \omega$ for all $x \in R, \omega \in \Omega$ and $\omega^{*} \in \Omega^{*}$;

(ii) $\left(\omega_{1}^{*}+\omega_{2}^{*}\right) \omega=\omega_{1}^{*} \omega+\omega_{2}^{*} \omega$ for all $\omega \in \Omega$ and $\omega_{1}^{*}, \omega_{2}^{*} \in \Omega^{*}$;

(iii) $\alpha^{*}\left(\omega_{1}+\omega_{2}\right)=\alpha^{*} \omega_{1}+\alpha^{*} \omega_{2}$ for all $\alpha^{*} \in \Lambda_{s}^{*}$ and $\omega_{1}, \omega_{2} \in \Omega$;

(iv) $\left(\omega \omega^{*}\right) \omega_{1}=\omega\left(\omega^{*} \omega_{1}\right)$ for all $\omega, \omega_{1} \in \Omega$ and $\omega^{*} \in \Omega^{*}$.

Proof. (i), (ii) and (iii). For all $\lambda \in \Lambda$ we have (i) $\lambda\left(\left(\omega^{*} x\right) \omega\right)=\left(\lambda\left(\omega^{*} x\right)\right) \omega=\left(\left(\lambda \omega^{*}\right) x\right) \omega=$ $\left(\lambda \omega^{*}\right)(x \omega)=\lambda\left(\omega^{*}(x \omega)\right)$ and

(ii) $\lambda\left(\left(\omega_{1}^{*}+\omega_{2}^{*}\right) \omega\right)=\left(\lambda\left(\omega_{1}^{*}+\omega_{2}^{*}\right)\right) \omega=\left(\lambda \omega_{1}^{*}+\lambda \omega_{2}^{*}\right) \omega=\left(\lambda \omega_{1}^{*}\right) \omega+\left(\lambda \omega_{2}^{*}\right) \omega=\lambda\left(\omega_{1}^{*} \omega+\omega_{2}^{*} \omega\right)$

(iii) $\lambda\left(\alpha^{*}\left(\omega_{1}+\omega_{2}\right)\right)=\left(\lambda \alpha^{*}\right)\left(\omega_{1}+\omega_{2}\right)=\left(\lambda \alpha^{*}\right) \omega_{1}+\left(\lambda \alpha^{*}\right) \omega_{2}=\lambda\left(\alpha^{*} \omega_{1}\right)+\lambda\left(\alpha^{*} \omega_{2}\right)=\lambda\left(\alpha^{*} \omega_{1}\right)+$ $\lambda\left(\alpha^{*} \omega_{2}\right)=\lambda\left(\alpha^{*} \omega_{1}+\alpha^{*} \omega_{2}\right)$ and the results follow.

(iv) Suppose $\omega=\sum \pm s_{i} \lambda_{i}^{\prime}$ with $s_{i} \in S$ and $\lambda_{i}^{\prime} \in \Lambda$. Then $\left(\omega \omega^{*}\right) \omega_{1}=\left(\left(\sum \pm s_{i} \hat{\lambda}_{i}^{\prime}\right) \omega^{*}\right) \omega_{1}=$ $\left(\sum \pm s_{i}\left(\lambda_{i}^{\prime} \omega^{*}\right)\right) \omega_{1}=\sum \pm s_{i}\left(\lambda_{i}^{\prime} \omega^{*}\right) \omega_{1}=\sum \pm s_{i}\left(\lambda_{i}^{\prime}\left(\omega^{*} \omega_{1}\right)\right)=\left(\sum \pm s_{i} \lambda_{i}^{\prime}\right)\left(\omega^{*} \omega_{1}\right)=\omega\left(\omega^{*} \omega_{1}\right)$.

Proposition 5.14. If $\bar{x} \in \bar{R}, \omega \in \Omega$ and $\omega^{*} \in \Omega^{*}$ we have

(i) $\bar{x}\left(\omega^{*} \omega\right)=\left(\bar{x} \omega^{*}\right) \omega$;

(ii) $\left(\omega^{*} \omega\right) \bar{x}=\omega^{*}(\omega \bar{x})$;

(iii) $\omega\left(\bar{x} \omega^{*}\right)=(\omega \bar{x}) \omega^{*}$.

Proof. (i) For $\lambda \in \Lambda$ let $\lambda \bar{x}=\sum a_{i} \lambda_{i}^{\prime}$ with $a_{i} \in R$ and $\lambda_{i}^{\prime} \in \Lambda$. Then $\lambda\left(\bar{x}\left(\omega^{*} \omega\right)\right)=$ $(\lambda \bar{x})\left(\omega^{*} \omega\right)=\left(\sum a_{i} \lambda_{i}^{\prime}\right)\left(\omega^{*} \omega\right)=\sum a_{i}\left(\lambda_{i}^{\prime}\left(\omega^{*} \omega\right)\right)=\sum a_{i}\left(\left(\lambda_{i}^{\prime} \omega^{*}\right) \omega\right)=\sum\left(a_{i}\left(\lambda_{i}^{\prime} \omega^{*}\right)\right) \omega=\left(\sum a_{i}\left(\lambda_{i}^{\prime} \omega^{*}\right)\right) \omega$ and $\lambda\left(\left(\bar{x} \omega^{*}\right) \omega\right)=\left(\lambda\left(\bar{x} \omega^{*}\right)\right) \omega=\left((\lambda \bar{x}) \omega^{*}\right) \omega=\left(\left(\sum a_{i} \lambda_{i}^{\prime}\right) \omega^{*}\right) \omega=\left(\sum a_{i}\left(\lambda_{i}^{\prime} \omega^{*}\right)\right) \omega$ and so (i) is proved.

(ii) $\lambda\left(\left(\omega^{*} \omega\right) \bar{x}\right)=\left(\lambda\left(\omega^{*} \omega\right)\right) \bar{x}=\left(\left(\lambda \omega^{*}\right) \omega\right) \bar{x}=\left(\lambda \omega^{*}\right)(\omega \bar{x})=\lambda\left(\omega^{*}(\omega \bar{x})\right)$ as $\bar{x}$ is an $R$ homomorphism of $\Omega$ and the result follows.

(iii) By definition of $\bar{x} \omega^{*}$ we have $\lambda\left(\bar{x} \omega^{*}\right)=(\lambda \bar{x}) \omega^{*}$ for all $\lambda \in \Lambda$ and consequently if $\omega=\sum a_{i} \lambda_{i}^{\prime}$ we have $\omega\left(\bar{x} \omega^{*}\right)=\left(\sum a_{i} \lambda_{i}^{\prime}\right)\left(\bar{x} \omega^{*}\right)=\sum a_{i}\left(\lambda_{i}^{\prime}\left(\bar{x} \omega^{*}\right)\right)=\sum a_{i}\left(\left(\lambda_{i}^{\prime} \bar{x}\right) \omega^{*}\right)=$ $\left(\sum a_{i}\left(\lambda_{i}^{\prime} \bar{x}\right)\right) \omega^{*}=\left(\left(\sum a_{i} \lambda_{i}^{\prime}\right) \bar{x}\right) \omega^{*}=(\omega \bar{x}) \omega^{*}$.

Theorem 5.15. (i) $\Omega . \Omega^{*}=R$;

(ii) $\Omega^{*} . \Omega=\bar{R}$.

Proof. (i) Trivial.

(ii) From the definition we have $\Omega^{*} \Omega \subseteq \bar{R}$. Now let $\bar{x} \in \bar{R}, \lambda \in \Lambda$ and $\lambda \bar{x}=\sum a_{i} \lambda_{i}^{\prime}$ with $a_{i} \in R$ and $\lambda_{i}^{\prime} \in \Lambda$. Define $\omega_{i}^{*}$ in $\Omega^{*}$ by $\lambda \omega_{i}^{*}=a_{i}$ and $\lambda^{\prime} \omega_{i}^{*}=0$ if $\lambda^{\prime} \neq \lambda$. Then $\bar{e}_{\lambda} \bar{x}=\sum \omega_{i}^{*} \lambda_{i}^{\prime} \in \Omega^{*} . \Omega$ for all $\lambda \in \Lambda$ and by the Corollary of Theorem 2 of [18] we have $\bar{x} \in \Omega^{*}, \Omega$. 
Let $\Omega_{1}, \Omega_{2}$ and $\Omega_{3}$ be $v(R,+)$-free left $(R, S)$-groups, $\bar{x}, \bar{y} \in \operatorname{Hom}_{R}\left(\Omega_{1}, \Omega_{2}\right)$ and $\bar{z} \in \operatorname{Hom}_{R}\left(\Omega_{2}, \Omega_{3}\right)$.

Definition 5.16. The dual map $\bar{x}^{*}: \Omega_{2}^{*} \rightarrow \Omega_{1}^{*}$ of $\bar{x}$ is defined by $\omega_{1}\left(\bar{x}^{*}\left(\omega_{2}^{*}\right)\right)=\left(\omega_{1} \bar{x}\right) \omega_{2}^{*}$ for all $\omega_{1} \in \Omega_{1}$ and $\omega_{2}^{*} \in \Omega_{2}^{*}$.

It is easily verified that $(\bar{x}+\bar{y})^{*}=\bar{x}^{*}+\bar{y}^{*}$ and $(\overline{x z})^{*}=\bar{x}^{*} \bar{z}^{*}$.

Let $\vec{R}^{*}$ denote the matrix d.g. near-ring over $R$ defined by Meldrum and Van der Walt. Since by Theorem 5.6 we have $\Omega^{*} \cong \bar{R}^{n}$ as d.g. right $(R, S)$-groups, we may consider the elements of $\bar{R}^{*}$ as elements of $\operatorname{Map}\left(\Omega^{*}, \Omega^{*}\right), f_{i j}^{r}$ as the element of $\operatorname{Map}\left(\Omega^{*}, \Omega^{*}\right)$ such that $f_{i j}^{r}\left(\sum_{k=1}^{n} \lambda_{k}^{*} r_{k}\right)=\lambda_{i}^{*} r r_{j}$ and $I^{n}$ as $\Omega^{*} . I$.

Theorem 5.17. $\bar{R}$ is near-ring isomorphic to $\bar{R}^{*}$.

Proof. Define $\psi: \bar{R} \rightarrow \operatorname{Map}\left(\Omega^{*}, \Omega^{*}\right)$ by $\psi(\bar{x})=\bar{x}^{*}$. Then $\psi$ is clearly a near-ring monomorphism. Further $\left(r \bar{e}_{\lambda_{i} \lambda_{j}}\right)^{*}=f_{i j}^{r}$ for all $r \in R$. Now by definition we have $\left\{f_{i j}^{r}: r \in R, 1 \leq i, j \leq n\right\}$ generates $\bar{R}^{*}$ and by Theorem 4.4 we have $\bar{R}$ is generated by the set $\left\{r \bar{e}_{\lambda_{j} \lambda_{j}}: r \in R, 1 \leq i, j \leq n\right\}$. Consequently we have $\psi(\bar{R})=\bar{R}^{*}$ and the result follows.

Acknowledgement. This research was done while the author was attached to the Tagajo Campus of the Tohoku Gakuin University and the author would like to acknowledge with gratitude the very generous help and kind hospitality accorded to him and his wife by Tohoku Gakuin University and in particular by Professor Shoji Kyuno and his family.

\section{REFERENCES}

1. S. J. AbbasI, Distributively generated matrix near-rings, Preprint 93/79, International Centre for Theoretical Physics, Trieste, Italy, 1993.

2. S. J. Abbasi and J. D. P. Meldrum, On matrix near-rings, Math. Pannon. 2 (1991), 107-113.

3. S. J. Abbasi, J. D. P. Meldrum and J. H. Meyer, The $J_{0}$-radical of matrix near-rings, Arch. Math. 56 (1991), 137-139.

4. J. C. Beidleman, On near-rings and near-ring modules (Doctoral dissertation, Pennsylvania State University, 1964).

5. A. FröHLICH, Distributively generated near-rings (I, Ideal Theory), Proc. London Math. Soc. (3) 8 (1958), 76-94.

6. A. Fröhlich, Distributively generated near-rings (II. Representation Theory), Proc. London Math. Soc. (3) 8 (1958), 95-108.

7. A. Fröhlich, On groups over a d.g. near-ring I, Quart. J. Math. Oxford Ser. (2) 11 (1960), 193-210.

8. A. FröhliCh, On groups over a d.g. near-ring II, Quart. J. Math. Oxford Ser. (2) 11 (1960), 211-228. 
9. H. E. Heatherly, Matrix near-rings, J. London Math. Soc. (2) 7 (1973), 355-356.

10. S. Ligh, A note on matrix near-rings, J. London Math. Soc. (2) 11 (1975), 383-384.

11. J. D. P. Meldrum, Near-rings and their links with groups (Research Notes in Mathematics, Pitman, 134, 1985).

12. J. D. P. Meldrum, Matrix near-rings, Contrib. Gen. Alg. 8 (ed. G. Pilz, Holder-PichlerTempsky, Wien 1992, 189-204).

13. J. D. P. Meldrum and J. H. Meyer, Intermediate ideals in matrix near-rings, Paper presented to the International Conference in Near-rings and Near-fields held at Hamburg in August, 1995.

14. J. D. P. Meldrum and A. P. J. VAN DeR WAlt, Matrix near-rings, Arch. Math. 47 (1986), 312-319.

15. J. H. MeYeR, Matrix near-rings (Ph.D. thesis, Stellenbosch University, South Africa, 1986).

16. Hanna Neumann, On varieties of groups and their associated near-rings, Math. $Z$. 65 (1956), 36-69.

17. V. Tharmaratinam, Complete primitive distributively generated near-rings, Quart. $J$. Math. Oxford Ser. (2) 18 (1967), 293-313.

18. V. Tharmaratinam, Endomorphism near-ring of a relatively free group, Math. $Z .113$ (1970), 119-135.

19. V. Tharmaratinam, Division d.g. near-rings, J. London Math. Soc. (2) 14 (1976), $135-147$.

20. V. Tharmaratinam, Division d.g. near-rings II, Bull. Austral. Math. Soc. 35 (1987), 73-80.

21. V. Tharmaratinam, Regular topological distributively generated near-rings, Bull. Austral. Math. Soc. 35 (1987), 59-72.

22. A. P. J. VAN DeR WALT, Primitivity in matrix near-rings, Quaestiones Math. 9 (1986), 459-469.

23. A. P. J. VAN DER WALT, On two sided ideals in matrix near-rings, in Near-rings and Near-fields (ed. G. Betsch, North Holland, Amsterdam, 1987), 267-272.

Department of Mathematics and Statistics

UNIVERSITY OF JAFFNA

THIRUNEL VEL Y, SRI LANKA 